\title{
High-Speed Railway Traction Power Supply Equipment Condition-Based Maintenance Decision Model Based on Abrasion Analysis
}

\author{
Hang Liu* and Qunzhan Li
}

School of Electrical Engineering, Southwest Jiaotong University, Chengdu, 610031, China

\begin{abstract}
According to abrasion process of machine equipment parts of overhead line system for traction power supply and characters of wear-out failures, considering on factor changes of all kinds of production conditions in practical operation, set permit of abrasion loss in coordinating with normal distribution, and abrasion loss of parts as random variables, and then establish the relation between maintenance costs and random variable of abrasion loss with the principle of lowest maintenance cost, thus concluding with the optimum maintenance abrasion value. Then the functional relation between abrasion loss and running time can be concluded with the gray model, thus the decision model in the optimum period with the lowest maintenance cost can be concluded.
\end{abstract}

Keywords: High-speed railway, traction power supply, overhead line system, maintenance decision.

\section{INTRODUCTION}

The high running speed of high-speed rail leads to strong vibration intensity and serious equipment abrasion. Abrasion is the significant problem faced by equipment functioning of high-speed rail overhead line system, equipment failure resulted from which can trigger interruption of power supply off and on, with serious influences generated which must be repaired or changed before equipment failure with precautions [1-3].

Since the occurrence of preventive maintenance system for equipment in 1960s, it has been superior to passive afterfailure maintenance in the aspect of its effect on modernized production, the researches on which have been hot in academic field [4]. Researchers have put forward numerous maintenance models, mostly with Markoff chain or half Markoff chain for researches on maintenance periods in long-time running of equipment. The data needed in the application of these maintenance models is in the nature of hysteresis, which may be difficult to be applied in maintenance scheduling [5]. Due to general occurrence of malfunction in functioning with uncertainty, dealing with dynamic problems with static methods is difficult. The pertinence and practicability of equipment maintenance are strong, so different equipment have different dominant failure patterns and different maintenance requirements; there are even obvious difference in failure modes of the completely same equipment due to difference in working environment and loads. As a result, the establishment of convenient, practical equipment maintenance decision model is necessary for directing field maintenance scheduling $[6,7]$.
According to statistics, the main factor of contracting lifetime of equipment parts for overhead line system is abrasion. Among the machine parts out of service, the amount of those, which are out of function due to abrasion, occupies $80 \%$, and abrasion can also trigger other failure modes [8][9]. However, it is the main failure mode that has not been established with simple and effective common calculation methods. Considering on microanalysis, the abrasion loss depends not only on unit run time, but also on many complicated factors like load, parts quality and service environment and so on, all of which make it difficult for gaining completed and common computational formula. On the other hand, most abrasion failures have obvious developmental process, belonging to gradual failure. The developmental rule of parts abrasion loss can be concluded through analysis on its developmental process, based on which, the preventive maintenance can be conducted.

\section{ABRASION ANALYSIS}

The abrasion process of machine and equipment parts can be generally divided into three stages displayed in Fig. (1) such as initial abrasion, normal abrasion and intensive abrasion. Abrasion is a continuous damaging process. The fit clearance among parts will be expanded with time and gradual increase of parts abrasion loss. When practical abrasion loss surpasses the abrasion loss permit, equipment malfunction or failure may be incurred. As to equipment maintenance, preventive maintenance should be adopted previous to approaching to the abrasion loss permit, as well as before the equipment malfunction, with parts exceeding abrasion loss permit repaired or changed $[10,11]$.

However the abrasion loss and abrasion lifetime of equipment parts are random variables, for that even the same kind of part working under the same condition are different in aspects of abrasion loss and time for certain abrasion loss, 


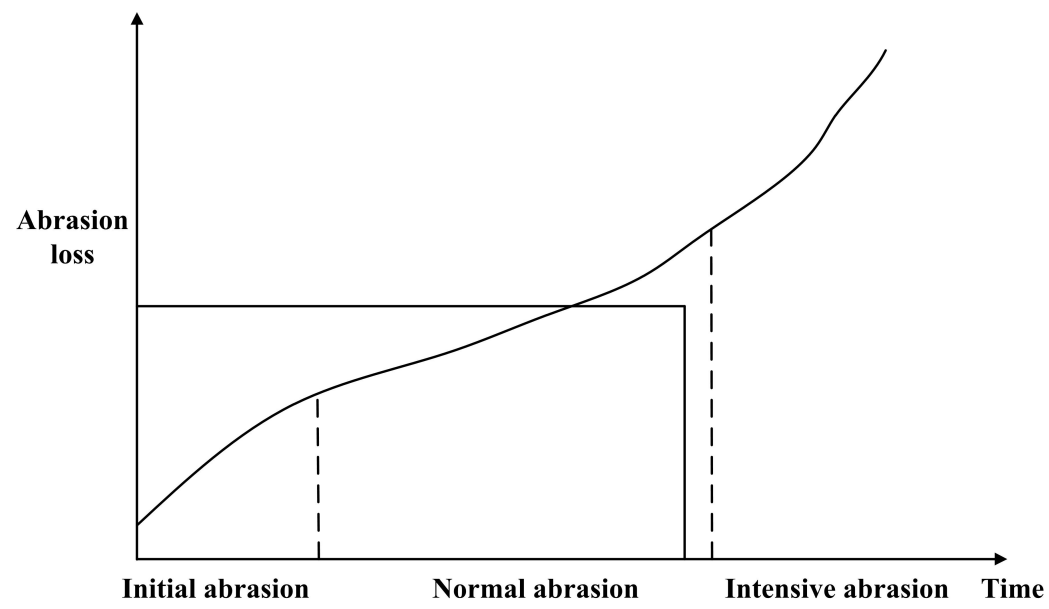

Fig. (1). Abrasion Process of Parts.

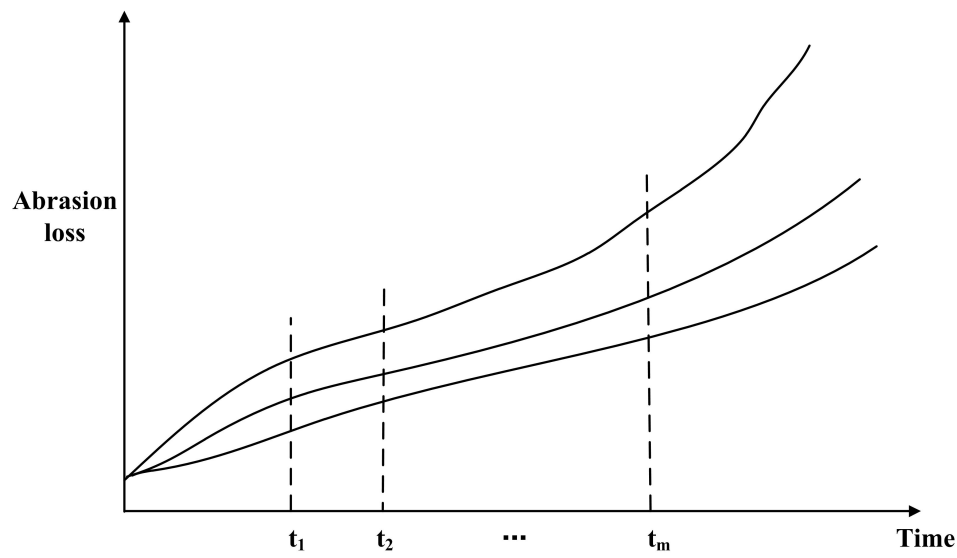

Fig. (2). Discreteness of Abrasion Loss.

and with the increase in working time and accumulated abrasion loss, the dispersion degree becomes larger and larger, displaying in Fig. (2). As a result, in the process of confirming abrasion loss permit of parts, a large amount of data needs to be collected for statistics.

\section{MAINTENANCE DECISION MODEL}

\subsection{Confirmation of Optimum Abrasion Loss}

In general, the key of maintenance decision is the confirmation of optimum maintenance periods T. Most existing models adopt running time $t$ as random variable and establish decision model according to rules with largest availability or lowest cost. The maintenance period concluded with this method is a fixed value T. This method is convenient for formulating working plan, but may be inaccurate in practical production. In practical work, under the influence of many factors like degree of functional stability of equipment, production conditions and so on, the abrasion process of parts seem uncertain with different abrasion loss in the same running time, as well as uncertain functional lifetime of parts. At this time, direct confirmation of maintenance period $\mathrm{T}$ as fixed value becomes infeasible. On the other hand, the abrasion malfunction or failure is resulted from abrasion loss exceeding permit value, which manes that no matter how complicated the functional process of parts is, only if the abrasion loss of parts approaches or reaches the abrasion loss permit, there is great possibility of the occurrence of malfunction. As a result, abrasion loss of parts $\mathrm{x}$ can be established as random variable with the principle of lowest maintenance cost, the decision method of maintenance period $\mathrm{T}$ can be concluded through confirmation of the optimum abrasion loss $\mathrm{x}$ with certain principle.

In this method, following situation can be set:

1) The abrasion loss complies with normal distribution.

2) Minor repair is adopted when malfunction occurs in maintenance period without changing equipment failure rate with restorer only. 


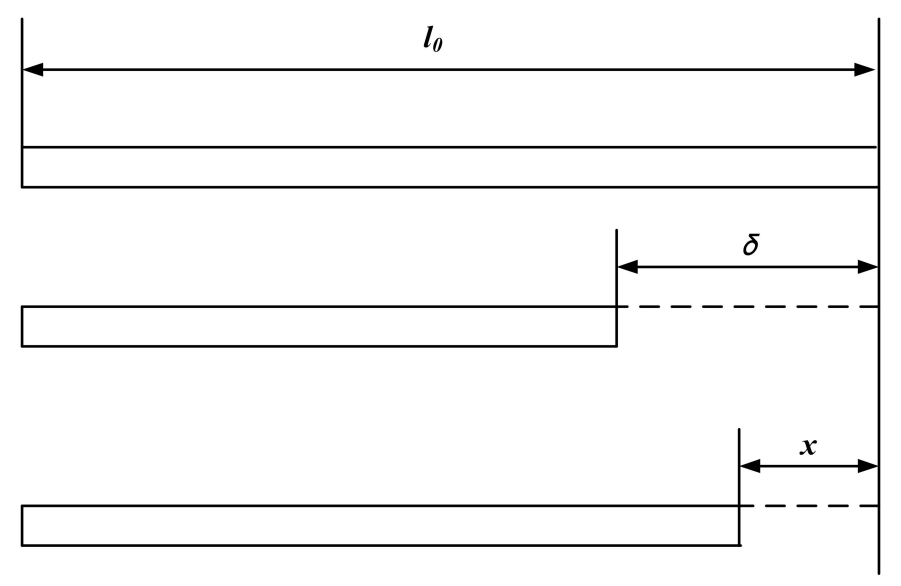

Fig. (3). Relation Between Quantity of Parts and Initial Size.

3) The comparison between time of minor repair and maintenance period can be neglected.

Fig. (3) display practical parts abrasion loss $\mathrm{x}$, abrasion loss permits $\delta$ and relation among original lengths of parts $l_{0}$. The distribution of abrasion loss permit $\delta$ is $\delta \sim \mathrm{N}\left(\delta_{0}, \sigma_{\delta}^{2}\right)$. The confirmation of $\delta_{0}, \sigma_{\delta}$ can be gained through collecting practical data by application of mathematical statistic method.

$$
\begin{aligned}
& p\left(\delta_{0}-x>0\right)=R(x)=\int_{x}^{+\infty} \frac{1}{\sqrt{2 \pi} \cdot \sigma_{\delta}} \exp \left(-\frac{\left(\delta_{0}-x\right)^{2}}{2 \sigma_{\delta}^{2}}\right) \cdot d t \\
& =\Phi\left(\frac{\delta_{0}-x}{\sigma_{\delta}}\right)=\Phi(z)
\end{aligned}
$$

$\Phi(z)$ is the corresponding percentage of standard normal distribution of $z$, so concluding with function expression of malfunction

$$
\lambda(x)=-\frac{R^{\prime}(x)}{R(x)}=-\frac{\Phi^{\prime}\left(\frac{\delta_{0}-x}{\sigma_{\delta}}\right)}{\Phi\left(\frac{\delta_{0}-x}{\sigma_{\delta}}\right)}
$$

Suppose during maintenance period $\mathrm{T}, \mathrm{c}_{1}$ is preventive maintenance expense (including production failure loss), $\mathrm{c}_{2}$ is the minor repair expense when malfunction occurring (including production failure loss). Set $\mathrm{N}(\mathrm{x})$ as the expected value for minor repair with abrasion loss being $\mathrm{x}$, then the anticipated value during maintenance period $\mathrm{C}(\mathrm{x})$ is

$C(x)=\frac{c_{2} N(x)+c_{1}}{x}$

$\mathrm{N}$ (x) equals to accumulated risk function under the equal condition, which is

$N(x)=\int_{0}^{x} \lambda(u) d u=-\ln R(x)$
Then conclude with

$C(x)=\frac{-c_{2} g \int_{0}^{x} \lambda(u) d u+c_{1}}{x}$

As to (5) differential, set it as 0 , conclude with,

$$
x \lambda(x)-\int_{0}^{x} \lambda(u) d u=\frac{c_{1}}{c_{2}}
$$

It is obvious that, if $\lambda(x)$ is monotone function, this formula has the only one conclusion as $x^{*}<\infty$. For that the abrasion loss is set to comply with normal distribution, concluded from literature that its malfunction rate $\lambda(\mathrm{x})$ is monotone rising function, so this formula has only one answer.

Introduce formula (4) into formula (6), conclude with

$-x \mathrm{~g} \frac{R^{\prime}(x)}{R(x)}+\ln R(x)=\frac{c_{1}}{c_{2}}$

\subsection{The Amount of Abrasion Measurement Model}

After concluding $\mathrm{x}$, the functional expression between abrasion loss and time $t$ needs to be confirmed.

Due to continuous process of abrasion, it can be expressed with the functional time t. however, due to uncertainty of abrasion process influenced by many factors in production, the analysis and calculation on abrasion loss of parts with the common methods like regression analysis, variance analysis in mathematical statistics may be difficult to deal with due to incomplete information. However, the grey system theory thinks that any random process is grey value changing within certain scale and certain time period, and regards random process as grey process. The grey system is to seek changing rules through systemization of original data, which is an approach to seek practical rules of data from data, which is called grey sequence generation. All grey sequence can generate weaken random with rules displaying. 
Table 1. Abrasion Loss.

\begin{tabular}{|c|c|c|c|c|}
\hline Serial No & Abrasion Loss (mm) & Frequency & Frequency (\%) & Accumulated Frequency \\
\hline \hline 1 & $2.00-2.09$ & 2 & 3.45 & 3.45 \\
\hline 2 & $2.10-2.19$ & 3 & 5.17 & 13.79 \\
\hline 3 & $2.20-2.29$ & 3 & 5.17 & 36.2 \\
\hline 4 & $2.30-2.39$ & 12 & 18.41 & 55.17 \\
\hline 5 & $2.40-2.49$ & 13 & 27.59 & 82.76 \\
\hline 6 & $2.50-2.59$ & 17 & 17.07 & 94.83 \\
\hline 7 & $2.60-2.69$ & 17 & 5.17 & 100.00 \\
\hline 8
\end{tabular}

Grey model mainly applies $\operatorname{GM}(1,1), \operatorname{GM}(1,1)$ models, which is to establish univariate constant coefficient differentiate equation, which is practically exponential model applicable to description of general energy system not changing relative to the environment, as a result, being grey model applicable to prediction. As the above description, abrasion process is a continuous process, and abrasion loss function is a monotone rising function, which comply with the modeling conditions of grey models.

$\mathrm{GM}(1,1)$ expression of model is:

$x^{(0)}(k)+a z^{(1)}(k)=b$

Of which $x^{(0)} \in X^{(0)}=\left(x^{(0)}(1), x^{(0)}(1), \cdots x^{(0)}(n)\right)$ is original nonnegative number sequence

$$
Z^{(1)}(k)=0.5 \sum_{i=1}^{k} x^{(0)}(i)+0.5 \sum_{i=1}^{k-1} x^{(0)}(i)
$$

$k=1,2, n$

Through winterization equation, the time response function of winterization equation $\frac{d(x)^{(1)}}{d t}+a x=b$ is

$x^{(1)}(t)=\left(x^{(1)}(0)-\frac{b}{a}\right) \exp (-a t)+\frac{b}{a}$

Conclude that the time expression of $\operatorname{GM}(1,1)$ is

$\hat{x}(k+1)=\left(x^{(1)}(0)-\frac{b}{a}\right) \exp (-a t)+\frac{b}{a}$

Restore the value concluded

$\hat{x}^{(0)}(k+1)=\hat{x}^{(1)}(k+1)-\hat{x}^{(1)}(k)$

When $\mathrm{k}>\mathrm{n}$, this model will predict the value of next time period. The application of this model in predicting abrasion process has gained satisfied effects. In practical application of this model, the abrasion tendency will change considering the changes of factors like production conditions, thus, a new data will be gained at any time and be introduced into X, with deduction of the oldest interest $\mathrm{x}^{(0)}(1)$. Establish metabolism model GM $(1,1)$, and keep the latest tendency of abrasion condition reflected through model and increase prediction precision.

After establishing functional expression between abrasion loss and time, maintenance $\mathrm{T}$ can be induced based on practical $\mathrm{x}$. the maintenance $\mathrm{T}$ concluded is changeable, which is more applicable to the practical production condition compared with previous results concluded through previous decision models.

\section{EXPERIMENT AND ANALYSIS}

The high functional speed of high-speed rail results in strong vibration intensity of equipment in overhead line system as well as serious abrasion of equipment [12][13]. Abrasion is the significant problem faced by equipment functioning of high-speed railway overhead line system. The malfunction of interruption of power supply triggered by equipment failure resulted from abrasion occurs off and on with serious influence generated. Preventive maintenance must be adopted for maintenance or changing before equipment failure. According to statistics, the main factor for contracting parts lifetime of overhead line system is abrasion. Among parts out of service, those failed due to abrasion occupy $60 \%$, and abrasion can trigger other failure patterns.

Gain 60 parts out of service in the same kind of overhead line system, measure the abrasion value, conclude with Table 1.

Conclude from data processing that the abrasion loss of this part out of service complies with normal distribution; abrasion loss permit $\delta_{0}$ and $\sigma_{\delta}$ can be gained with application of optimum current method of estimation without deviation:

$$
\delta_{0}=0.24572, \sigma_{\delta}^{2}=0.02733, \sigma_{\delta}=0.1721
$$


Table 2. Abrasion Loss Records of Parts in Function.

\begin{tabular}{|c|c|c|c|c|}
\hline Time t/h & $\mathbf{1 0}$ & $\mathbf{1 5}$ & $\mathbf{2 0}$ & $\mathbf{2 5}$ \\
\hline \hline Sequence $(\mathrm{k})$ & 1 & 2 & 3 & 4 \\
\hline Abrasion loss $(\mathrm{mm})$ & 1.0 & 1.27 & 1.41 & 1.53 \\
\hline
\end{tabular}

Table 3. Simulation Value of Abrasion Loss of Parts.

\begin{tabular}{|c|c|c|c|c|}
\hline $\mathbf{k}$ & $\mathbf{1}$ & $\mathbf{2}$ & $\mathbf{3}$ & $\mathbf{4}$ \\
\hline \hline$\hat{x}(k)$ & 1.0 & 1.28 & 1.39 & 0.157 \\
\hline
\end{tabular}

Table 4. Comparisons Between True Value of Abrasion Loss of Parts and Error of Simulation Value.

\begin{tabular}{|c|c|c|c|}
\hline $\mathbf{k}$ & $\mathbf{1}$ & $\mathbf{2}$ & $\mathbf{3}$ \\
\hline \hline$\varepsilon(k)=x^{(0)}(k)-\hat{x}^{(0)}(k)$ & 0 & 0.001 & 0.001 \\
\hline$\Delta k=\left|\frac{\varepsilon(k)}{x^{(0)}(k)}\right|$ & 0 & $0.8 \%$ & $1.43 \%$ \\
\hline
\end{tabular}

Suppose $c_{1} / c_{2}=1 / 4$, substitute into (7), conclude with $\mathrm{x}^{*} \approx 2.08 \mathrm{~mm}$.

Collect abrasion loss of parts of this kind of equipment in functional process, display like Table 2.

Establish GM $(1,1)$ makes it winterized, conclude with time response expression:

$$
\hat{x}(k+1)=1.372 \exp (0.08996 k)-1.232
$$

Calculate with value of simulation based on this formula, like Table 3.

Residual and relative error as to original value is like Table 4:

The formula can be applied for prediction with small error, introduce $\mathrm{x}$ into the formula and conclude with maintenance period of parts as $\mathrm{T}=49$ weeks, conforming to the practical situation of parts functioning.

\section{CONCLUSION}

According to abrasion process of machine equipment parts of overhead line system for traction power supply and characters of wear-out failures, considering on factor changes of all kinds of production conditions in practical operation, set permit of abrasion loss in coordinating with normal distribution, and abrasion loss of parts as random variables, and then establish the relation between maintenance costs and random variable of abrasion loss with the principle of lowest maintenance cost, thus concluding with the optimum maintenance abrasion value. Then the functional relation between abrasion loss and running time can be concluded with the gray model, thus the decision model in the optimum period with the lowest maintenance cost can be concluded.

\section{CONFLICT OF INTEREST}

The authors confirm that this article content has no conflicts of interest.

\section{ACKNOWLEDGEMENTS}

This research is supported by 2014 China Railway Corporation Technology Research and Development Project.

\section{REFERENCES}

[1] Cao Jianyou, Traction Power Supply System for Electrified Railway, Beijing, China Railway Press, 1983 (in Chinese)

[2] Li Qunzhan, He Jianmin, Analysis on Traction Power Supply System, Southwest Jiaotong University Press, 2007.9(in Chinese)

[3] Wu Jiqin, Pantograph and Overhead Line System, Southwest Jiaotong University 2010.12:305(in Chinese)

[4] Jia Xisheng, Maintenance Decision Model Centered on Reliability, National Defense Press, 2007.1 (in Chinese)

[5] Ren Yan Jiang, D.N.Prab Murthy, Maintenance Management Decision Model, Science Press, 2008.6

[6] Ho, T.K., Chi, Y.L., Ferreial, L., Leung, K.K., and Siu, L.K., "Evaluation of maintenance schedules on railway traction power system", Proceeding of the Institution of Mechanical Engineers, Part F: J. Rail and Rapid Transit, 2006, 220(2): 91-102

[7] Yang Qinghe, Li Zhanling, Zhangsihai, "Development of high speed railway traction power supply computer repair management system", Railway Computer Application, 2011, Vol20 No.8. (in Chinese)

[8] Lin Wei, A class of Generalized Gradient abrasion Model for reliability analysis and repair strategy research Southwest Jiaotong University 2012.5 (in Chinese) 
[9] Lizhen, "Engineering machinery and equipment maintenance and repair of early abrasion", Technology and business, 2013.10(in Chinese)

[10] Yu Wanju, High-speed Electrified Railway Overhead Line System, Southwest Jiaotong University Press, 2003 (in Chinese).

[11] Qi Guangfeng, Research on Reliability and Maintenance of Highspeed Railway Overhead Line System, Southwest Jiaotong University Press, 2012.12 (in Chinese)
[12] Liu Zaimin, Research and Practice of Overhead Line System Maintenance Management System, railway locomotives, 2007,03:66-67. (in Chinese)

[13] He Zhengyou, Cheng Hongbo, "Research on Healthy Management of High-speed Railway Traction Power Supply System and Preventive System of Malfunction", power grid technology, 2012,10:259264. (in Chinese)

(C) Liu and Li; Licensee Bentham Open.

This is an open access article licensed under the terms of the Creative Commons Attribution Non-Commercial License (http://creativecommons.org/licenses/by-nc/3.0/) which permits unrestricted, non-commercial use, distribution and reproduction in any medium, provided the work is properly cited. 